\title{
ERGODIC CURRENTS DUAL TO A REAL TREE
}

\author{
THIERRY COULBOIS, ARNAUD HILION
}

\begin{abstract}
Let $T$ be an $\mathbb{R}$-tree with dense orbits in the boundary of Outer space. When the free group $\mathbb{F}_{N}$ acts freely on $T$, we prove that the number of projective classes of ergodic currents dual to $T$ is bounded above by $3 N-5$.

We combine Rips induction and splitting induction to define unfolding induction for such an $\mathbb{R}$-tree $T$. Given a current $\mu$ dual to $T$, the unfolding induction produces a sequence of approximations converging towards $\mu$.

We also give a unique ergodicity criterion.
\end{abstract}

\section{INTRODUCTION}

1.1. Main results. Let $\mathbb{F}_{N}$ be the free group with $N$ generators. M. Culler and K. Vogtmann CV86 introduced Outer space: the space $\mathrm{CV}_{N}$ of projective classes of free minimal actions of $\mathbb{F}_{N}$ by isometries on simplicial metric trees. We denote by $\mathrm{cv}_{N}$ the space of free minimal actions of $\mathbb{F}_{N}$ by isometries on simplicial metric trees: $\mathrm{cv}_{N}$ is the unprojectivised Outer space. The space $\mathrm{CV}_{N}$ is a contractible simplicial complex, with missing faces. The maximal dimension of a simplex in $\mathrm{CV}_{N}$ is $3 N-4$. Outer space admits a Thurston boundary, denoted by $\partial \mathrm{CV}_{N}$, which gives rise to a compactification $\overline{\mathrm{CV}}_{N}=\mathrm{CV}_{N} \uplus \partial \mathrm{CV}{ }_{N}$ of $\mathrm{CV}_{N}$. This compactification consists of projective classes of $\mathbb{R}$-trees with a minimal, very small action of $\mathbb{F}_{N}$ by isometries CL95, BF95. Again, we denote by $\partial \mathrm{cv}_{N}$ and $\overline{\mathrm{cv}}_{N}$ the corresponding unprojectivized spaces.

The group $\operatorname{Out}\left(\mathbb{F}_{N}\right)$ of outer automorphisms of the free group $\mathbb{F}_{N}$ acts on $\mathrm{CV}_{N}$. One basically considers that the Outer space plays the same role for $\operatorname{Out}\left(\mathbb{F}_{N}\right)$ as the Teichmüller space of a surface $S$ for the mapping class group $\mathrm{MCG}(S)$ of $S$ - see for instance [BV06] where the analogy is carried on.

Another space on which $\operatorname{Out}\left(\mathbb{F}_{N}\right)$ naturally acts, and which appears to capture slightly different informations from $\operatorname{Out}\left(\mathbb{F}_{N}\right)$, is the space $\mathbb{P} \operatorname{Curr}\left(\mathbb{F}_{N}\right)$ of projective classes of currents [Kap06, Kap05]. Let us recall what a current is.

The space $\partial \mathbb{F}_{N}$ of ends of the free group $\mathbb{F}_{N}$ is a Cantor set, equipped with an action by homeomorphisms of $\mathbb{F}_{N}$. The space $\partial \mathbb{F}_{N}$ is also the Gromov boundary of $\mathbb{F}_{N}$. The action of $\mathbb{F}_{N}$ on itself by left multiplication extends continuously to $\partial \mathbb{F}_{N}$. We denote by $\partial^{2} \mathbb{F}_{N}=\left(\partial \mathbb{F}_{N}\right)^{2} \backslash \Delta$ the double boundary of $\mathbb{F}_{N}$, where $\Delta$ stands for the diagonal. The double boundary inherits a product topology from $\partial \mathbb{F}_{N}$, and the action of $\mathbb{F}_{N}$ on $\partial \mathbb{F}_{N}$ gives rise to a diagonal action on $\partial^{2} \mathbb{F}_{N}$. The involution $(X, Y) \mapsto(Y, X)$ of $\partial^{2} \mathbb{F}_{N}$ is called the flip map. A current $\mu$ is a $\mathbb{F}_{N}$-invariant, flip-invariant, Radon measure (that is to say a Borel measure which is finite on compact sets) on $\partial^{2} \mathbb{F}_{N}$. We notice that a linear combination, with non-negative coefficients, of currents is still a current.

The space $\operatorname{Curr}\left(\mathbb{F}_{N}\right)$ of currents of $\mathbb{F}_{N}$ is equipped with the weak-*-topology: it is a locally compact space. The group $\operatorname{Out}\left(\mathbb{F}_{N}\right)$ naturally acts on $\operatorname{Curr}\left(\mathbb{F}_{N}\right)$ - see for instance $\operatorname{Kap} 06$ for a detailed description of this action. The space $\mathbb{P C u r r}\left(\mathbb{F}_{N}\right)$ of projective classes of (non-zero) currents equipped with the quotient topology is a compact set, and the action of $\operatorname{Out}\left(\mathbb{F}_{N}\right)$ on $\operatorname{Curr}\left(\mathbb{F}_{N}\right)$ induces an action on $\mathbb{P C u r r}\left(\mathbb{F}_{N}\right)$.

The spaces $\operatorname{Curr}\left(\mathbb{F}_{N}\right)$ and $\mathrm{cv}_{N}$ can be viewed, to some extent, as dual spaces. Indeed, M. Lustig and I. Kapovich [KL09] defined a kind of duality bracket:

$$
\begin{array}{ccc}
\langle\cdot, \cdot\rangle: \quad \overline{\operatorname{cv}}_{N} \times \operatorname{Curr}\left(\mathbb{F}_{N}\right) & \rightarrow \mathbb{R}_{\geq 0} \\
(T, \mu) & \mapsto\langle T, \mu\rangle .
\end{array}
$$

This bracket is characterized as being the unique continuous $\operatorname{Out}\left(\mathbb{F}_{N}\right)$-equivariant map $\overline{\operatorname{cv}}_{N} \times \operatorname{Curr}\left(\mathbb{F}_{N}\right) \rightarrow$ $\mathbb{R}_{\geq 0}$ which is $\mathbb{R}_{\geq 0}$-homogeneous in the first entry and $\mathbb{R}_{\geq 0}$-linear in the second one, and which satisfies that

Date: May 18, 2018.

The authors are supported by the grant ANR-10-JCJC 01010 of the Agence nationale de la recherche. 
for all $T \in \overline{\mathrm{cv}}_{N}$ and for all rational current $\mu_{w}$ induced by a conjugacy class of a primitive element $w$ of $\mathbb{F}_{N}$, $\left\langle T, \mu_{w}\right\rangle$ is the translation length of the conjugacy class of $w$ in $T$ - see [KL09]. The number $\langle T, \mu\rangle$ is the intersection number of $T$ and $\mu$.

A tree $T \in \overline{\mathrm{cv}}_{N}$ and a current $\mu \in \operatorname{Curr}\left(\mathbb{F}_{N}\right)$ are dual when $\langle T, \mu\rangle=0$. We notice that the nullity of the intersection number $\langle T, \mu\rangle=0$ only depends on the projective classes of $T$ and $\mu$. The main goal of this paper is to explain how to build all the currents dual to a given tree. We remark that the set of projective classes of currents dual to a given tree $T$ is convex: the extremal points of this set are ergodic currents.

Theorem 1.1. Let $T$ be an $\mathbb{R}$-tree with a free, minimal action of $\mathbb{F}_{N}$ by isometries with dense orbits. There are at most $3 N-5$ projective classes of ergodic currents dual to $T$.

The duality between trees and currents can also be unterstood by considering laminations. An $\mathbb{R}$-tree $T$ with an action of $\mathbb{F}_{N}$ by isometries with dense orbits has a dual lamination $L(T)$ CHL08b. I. Kapovich and M. Lustig [KL10 proved that a current is dual to $T$ if and only if it is carried by the dual lamination. Thus we can rephrase our main Theorem to:

Theorem 1.2. Let $T$ be an $\mathbb{R}$-tree with a free, minimal action of $\mathbb{F}_{N}$ by isometries with dense orbits. The dual lamination $L(T)$ carries at most $3 N-5$ projective classes of ergodic currents.

1.2. The surface case. Generally speaking, the present work is inspired by the situation of hyperbolic surfaces which we recall here.

Let $S=S_{g}$ be an oriented surface of genus $g$ with negative Euler characterisic $\chi(S)=2-2 g<0$. The mapping class group of $S$ acts on Teichmüller space $\operatorname{Teich}(S)$ which is homeomorphic to a ball of dimension $6 g-6$. Teichmüller space can be compactified by adding its Thurston boundary $\partial \mathrm{Teich}(S)$ which is homeomorphic to a sphere of dimension $6 g-7$. There are several useful models to describe $\partial$ Teich $(S)$, see for instance [Pau88]. In particular, points in $\partial \operatorname{Teich}(S)$ can be seen alternatively as projective classes of:

- $\mathbb{R}$-trees with a minimal small action by isometries of the fundamental group of the surface,

- measured geodesic laminations on $S$,

- measured singular foliations on $S$ (up to Whitehead equivalence).

- currents (see [Bon88)

A geodesic lamination may carry more than one transverse measure (even if the lamination is minimal). The simplex of measures carried by a given geodesic lamination embeds in the boundary of Teichmüller space and thus has dimension less than $6 g-5$. In fact, G. Levitt Lev83 proved that an orientable foliation carries at most $3 g-3$ distinct projective ergodic measures, see also Pap86.

It is not easy to exhibit a non-uniquely ergodic minimal foliation. Examples come from interval exchange transformations (IET). Indeed, the mapping torus of an IET gives rise to a foliated surface (through zippering process). H. Keynes and D. Newton [KN76] and M. Keane [Kea77] gave examples of non-uniquely ergodic minimal IET (and thus of non-uniquely ergodic minimal foliations on a surface). E. Sataev [Sat75] constructed minimal IET with exactly $k$ ergodic measures for $1 \leq k \leq 2 g$. The main tool to investigate ergodic properties of the foliation of an IET is to use a suitable induction such as Rauzy-Veech induction.

In the case of Outer space, there are fewer models available to describe points in the boundary $\partial \mathrm{CV}_{N}$. In particular, I. Kapovich and M. Lustig [KL07] proved that there is no Out $\left(\mathbb{F}_{N}\right)$-equivariant continuous embedding of $\partial \mathrm{CV}_{N}$ in the space of currents. Although D. Gaboriau and G. Levitt GL95] proved that the dimension of $\partial \mathrm{CV}_{N}$ is $3 N-5$ (see also V. Guirardel Gui00] where this result is reinterpreted in terms of length measures), we cannot deduce from this fact a bound on the number of ergodic currents dual to an $\mathbb{R}$-tree. This leads us to follow the alternative strategy of analysing the ergodic properties via an induction.

1.3. Outline of the proof. The general strategy to prove Theorem 1.1 consists in mimicking what has been done for interval exchange transformations. However, a tree in $\partial \mathrm{cv}_{N}$ is not necessarily transverse to the foliation of an interval exchange transformation. Thus we cannot use the Rauzy-Veech induction. Instead, for an $\mathbb{R}$-tree $T$ in $\partial \mathrm{CV}_{N}$ with dense orbits and a basis $A$ of $\mathbb{F}_{N}$, we defined, together with M. Lustig [CHL09], a kind of a band complex $S_{A}=\left(K_{A}, A\right)$ : the mapping torus of the system of isometries on the compact heart given by $A$. For such a band complex we can use the unfolding induction which consists in either the Rips induction [CH14] or the splitting induction [CHR11].

First, let us recall that a current can be seen as a Kolmogorov function. Given a graph $\Gamma$ and a marking isomorphism $\mathbb{F}_{N} \stackrel{\sim}{\rightarrow} \pi_{1}(\Gamma)$, the universal cover $\widetilde{\Gamma}$ is a (simplicial) tree with an action of $\mathbb{F}_{N}$. Points in $\partial \mathbb{F}_{N}$ 
correspond to points in the boundary of $\partial \widetilde{\Gamma}$ and elements $(X, Y) \in \partial^{2} \mathbb{F}_{N}$ are represented by bi-infinite lines in $\widetilde{\Gamma}$. The topology on $\partial^{2} \mathbb{F}_{N}$ is given by the cylinders: sets of lines that share a common subpath. Currents are completely described by the measures of the cylinders: For a current $\mu$ and a finite path $\gamma$ in $\Gamma$ (or rather its lift in $\widetilde{\Gamma})$, the Komolgorov function associated to $\mu$, assigns to $\gamma$ the measure $\mu(\gamma)$ of the cylinder defined by $\gamma$. (Note that the fuzzyness about the absence of base-point in the marking isomorphism or the choice of a lift of $\gamma$ in $\widetilde{\Gamma}$ is justified by the $\mathbb{F}_{N}$-invariance of currents.)

For a marked graph $\Gamma$ and a current $\mu$ we consider the non-negative vector $\mu_{\Gamma}=(\mu(e))_{e \in E(\Gamma)}$ of the $\mu$ measure of the cylinders defined by the edges of $\Gamma$. The unfolding induction, starting from an indecomposable $\mathbb{R}$-tree $T$ in $\partial \mathrm{CV}_{N}$, produces a sequence $\left(\Gamma_{n}\right)$ of marked graphs and a sequence of non-negative integer matrices $\left(M_{n}\right)$ such that for any current $\mu$ dual to $T$ :

(1) the sequence of vectors $\left(\mu_{\Gamma_{n}}\right)$ completely determines $\mu$,

(2) $\mu_{\Gamma_{n}}=M_{n} \mu_{\Gamma_{n+1}}$.

Using properties (11) and (2), we derive that the bound on the the dimension of the simplex of currents dual to $T$ is given by the Euler characteristic of the graphs $\Gamma_{n}$ which remains constant through splitting induction.

In fact, the sequence of marked graphs $\left(\Gamma_{n}\right)$ given by the unfolding induction limits to the support of the currents dual to $T$. It also gives a decomposition of the cylinders of the dual lamination $L(T)$ defined by the edges of $\Gamma_{n}$ similar to the Kakutani-Rokhlin towers approximations of a dynamical system - see for instance [Dur10, Definition 6.4.1] .

In Section 6] we adress the question of unique ergodicity: an $\mathbb{R}$-tree in $\partial \mathrm{CV}_{N}$ is uniquely ergodic if it is dual to a unique projective current. In the context of IET, there is a famous sufficient condition known as Masur criterion Mas82. This criterion can be understood using the sequence of matrices of the Rauzy-Veech induction, see for instance [Yoc05]. We derive such a criterion for $\mathbb{R}$-trees in $\partial \mathrm{CV}_{N}$ in terms of the sequence of matrices of the splitting induction.

Acknowledgements. We would like to thank John Smillie and Gilbert Levitt for giving us key references and Hossein Namazi and Alexandra Pettet for pointing out mistakes in previous versions.

\section{Preliminaries}

2.1. Trees and dual laminations. An $\mathbb{R}$-tree $T$ is a 0 -hyperbolic metric space. It has a Gromov boundary $\partial T$. We denote by $\widehat{T}=\bar{T} \cup \partial T$ the union of the metric completion of $T$ and its Gromov boundary. This is a topological space which is not compact in general. A direction $d$ at a point $P$ in $\widehat{T}$ is a connected component of $\widehat{T} \backslash\{P\}$. We weaken the topology on $\widehat{T}$ by considering the set of directions as a sub-basis of open sets, we denote by $\widehat{T}^{\text {obs }}$ the resulting topological space which is Hausdorff, compact and has exactly the same connected subsets as $\widehat{T}$ [CHL07. Indeed $\widehat{T}^{\text {obs }}$ is a dendrite in the terminology of B. Bowditch Bow99.

We denote by $\partial \mathbb{F}_{N}$ the Gromov-boundary of $\mathbb{F}_{N}: \partial \mathbb{F}_{N}$ is a Cantor set. Fixing a basis $A$ of $\mathbb{F}_{N}$, elements of $\mathbb{F}_{N}$ are finite reduced words in $A^{ \pm 1}$ and elements of $\partial \mathbb{F}_{N}$ are infinite reduced words in $A^{ \pm 1}$. Let $T$ be an $\mathbb{R}$ tree in $\partial \mathrm{cv}_{N}$ with dense orbits. For a point $P$ in $T$ the orbit map $\mathbb{F}_{N} \rightarrow T, u \mapsto u P$, has a unique continuous extension to a map $\mathcal{Q}: \partial \mathbb{F}_{N} \rightarrow \widehat{T}^{\text {obs }}$. This map $\mathcal{Q}$ does not depend on the choice of $P$ [LL03, LL08, CHL07].

We denote by $\partial^{2} \mathbb{F}_{N}=\left(\partial \mathbb{F}_{N}\right)^{2} \backslash \Delta$ the double boundary of $\mathbb{F}_{N}$, where $\Delta$ stands for the diagonal. The dual lamination CHL08a, CHL08b of the tree $T$ is

$$
L(T)=\left\{(X, Y) \in \partial^{2} \mathbb{F}_{N} \mid \mathcal{Q}(X)=\mathcal{Q}(Y)\right\} .
$$

This is a closed, $\mathbb{F}_{N}$-invariant, flip-invariant, subset of $\partial^{2} \mathbb{F}_{N}$.

The map $\mathcal{Q}$ induces a continuous [CHL09] map $\mathcal{Q}^{2}: L(T) \rightarrow \bar{T}$ (here the topology on $\bar{T}$ is the metric topology). The limit set $\Omega=\mathcal{Q}^{2}(L(T))$ of $T$ is the image of the lamination by $\mathcal{Q}$. The tree $T$ is of Levitt type if the limit set is totally disconnected.

2.2. Compact heart. Let $A$ be a basis of $\mathbb{F}_{N}$. Elements of $\mathbb{F}_{N}$ are vertices of the Cayley graph of $\mathbb{F}_{N}$ with respect to the basis $A$. Elements of $\partial \mathbb{F}_{N}$ are infinite reduced paths starting at 1 . Elements of $\partial^{2} \mathbb{F}_{N}$ are identified with bi-infinite reduced paths in the Cayley graph indexed by $\mathbb{Z}$. The unit cylinder of $\partial^{2} \mathbb{F}_{N}$ is the set of bi-infinite reduced paths going through 1 at index 0 :

$$
C_{A}(1)=\left\{(X, Y) \in \partial^{2} \mathbb{F}_{N} \mid X_{0} \neq Y_{0}\right\}
$$


where $X_{0}$ is the first letter of the infinite word $X$. The unit cylinder $C_{A}(1)$ is a compact set. We denote by $L_{A}(T)=L(T) \cap C_{A}(1)$ the symbolic lamination relative to $A$. It is a compact set. Its image by $\mathcal{Q}^{2}$ is the compact limit set relative to $A$ :

$$
\Omega_{A}=\mathcal{Q}^{2}\left(L_{A}(T)\right)=\mathcal{Q}^{2}\left(L(T) \cap C_{A}(1)\right) \subseteq \bar{T} .
$$

The convex hull of $\Omega_{A}$ is the compact heart $K_{A} \subseteq \bar{T}$ of the tree $T$ relative to $A$.

For each element $a \in A$ of the basis we consider its restriction to $K_{A}$ as a partial isometry. By allowing inverses and composition we get a pseudo-action of $\mathbb{F}_{N}$ on $K_{A}$. We denote by $S_{A}=\left(K_{A}, A\right)$ this system of isometries [CHL09].

An element $u$ of $\mathbb{F}_{N}$ is admissible if it is non-empty as a partial isometry. An infinite reduced word $X \in \partial \mathbb{F}_{N}$ is admissible if all its prefixes are admissible. In this case the domains of the prefixes form a nested sequence of non-empty compact subtrees of the compact heart $K_{A}$ and the domain of $X$ is their intersection, it is exactly the image of $X$ by the map $\mathcal{Q}$ :

$$
\{\mathcal{Q}(X)\}=\operatorname{dom} X=\bigcap_{n \in \mathbb{N}} \operatorname{dom}\left(X_{n}\right)
$$

where $X_{n}$ is the prefix of length $n$. A bi-infinite reduced word $Z$ has two halves and we write $Z=\left(Z_{-}, Z_{+}\right) \in$ $C_{A}(1)$. It is admissible if all its finite factors are admissible, or equivalently, if its two halves are admissible with the same domain. In this case, the domain of $Z$ is the common domain of its two halves:

$$
\operatorname{dom}(Z)=\{\mathcal{Q}(Z)\}=\left\{\mathcal{Q}\left(Z_{+}\right)\right\}=\left\{\mathcal{Q}\left(Z_{-}\right)\right\} .
$$

The set of admissible bi-infinite words of the system of isometries $S_{A}=\left(K_{A}, A\right)$ is the admissible lamination $L\left(S_{A}\right)$. This is a shift-invariant symmetric closed subset of the full-shift of bi-infinite reduced words in $A^{ \pm 1}$. We remark that a bi-infinite reduced word $Z$ corresponds to a pair $\left(Z_{-}, Z_{+}\right)$in $\partial^{2} \mathbb{F}_{N}$, and for a pair $(X, Y) \in \partial^{2} \mathbb{F}_{N}$, we can define the bi-infinite reduced word $X^{-1} Y$. This correspondance is rather between a shift-orbit of bi-infinite reduced words in $A^{ \pm 1}$ and an $\mathbb{F}_{N}$-orbit in $\partial^{2} \mathbb{F}_{N}$. By abuse of notations, using the above correspondance, we have:

Proposition 2.1 ([CHL09]). Let $T$ be an $\mathbb{R}$-tree with a minimal, very small action of $\mathbb{F}_{N}$ by isometries with dense orbits. Let $A$ be a basis of $\mathbb{F}_{N}$ and let $S_{A}=\left(K_{A}, A\right)$ be the associated system of isometries.

Then, the admissible lamination of $S_{A}$ is equal to the dual lamination of $T: L\left(S_{A}\right)=L(T)$.

For a word $w \in \mathbb{F}_{N}$, the cylinder $C_{A}(w) \subseteq L\left(S_{A}\right)$ is the set of bi-infinite admissible words $Z$ in $S_{A}$ which reads $w$ at index $0: w$ is a prefix of the positive half $Z_{+}$. The shifts of the cylinders form a sub-basis of open sets of the admissible lamination.

2.3. Systems of isometries. More generally a system of isometries $S=(F, A)$ is a compact forest $F$ together with a finite set $A$ of partial isometries of $F$. Here a compact forest is a disjoint union of finitely many compact $\mathbb{R}$-trees. A partial isometry is a non-empty isometry between two compact subtrees. The graph $\Gamma=\Gamma(S)$ of such a system of isometries has the connected components of $F$ as vertices and the partial isometries in $A$ as edges. The edge $a \in A$ goes from the connected component that contains its domain to the connected component of that contains its image. We always assume that $\Gamma$ is connected.

A finite reduced path $\gamma$ in $\Gamma$ is admissible if it is non-empty as a partial isometry. Its domain $\operatorname{dom}(\gamma)$ is a non-empty compact subtree of $F$. The infinite reduced path $X$ in $\Gamma$ is admissible if all its subpaths are admissible. Its domain $\operatorname{dom}(X)$ is the intersection of the nested domains of the initial subpaths of $X$. A bi-infinite reduced path $Z=\cdots Z_{-1} Z_{0} Z_{1} Z_{2} \cdots$ has to halves $Z_{-}=Z_{0}^{-1} Z_{-1}^{-1} \cdots$ and $Z_{+}=Z_{1} Z_{2} \cdots$. It is admissible if all its subpaths are admissible or equivalently if its two halves are admissible and its domain $\operatorname{dom}(Z)=\operatorname{dom}\left(Z_{-}\right) \cap \operatorname{dom}\left(Z_{+}\right)$is a non-empty compact subtree of $F$.

The admissible lamination $L(S)$ of the system of isometries $S=(F, A)$ is the set of bi-infinite admissible paths in $\Gamma$. For a finite reduced path $\gamma$ in $\Gamma$, the cylinder $C(\Gamma, \gamma)$ is the set of bi-infinite admissible paths $Z$ that goes through $\gamma$ at index $0: \gamma$ is a prefix of the positive half $Z_{+}$.

Following D. Gaboriau Gab97, a system of isometries has independent generators if the domain $\operatorname{dom}(X)$ of any infinite admissible path $X$ in $\Gamma$ contains a single point which we denote by $\mathcal{Q}(X)$. 
2.4. Currents. A current for the free group $\mathbb{F}_{N}$ is an $\mathbb{F}_{N}$-invariant, flip-invariant, Radon measure (that is to say a Borel measure which is finite on compact sets) on $\partial^{2} \mathbb{F}_{N}$. The support of a current is a lamination.

Let $T$ be an $\mathbb{R}$-tree with a minimal very small action of $\mathbb{F}_{N}$ by isometries with dense orbits. We denote by $\operatorname{PCurr}(T)$ the simplex of currents carried on the dual lamination $L(T)$. Let $A$ be a basis of $\mathbb{F}_{N}$ and $S_{A}=$ $\left(K_{A}, A\right)$ be the system of isometries on the compact heart of $T$. Recall that we identify the dual lamination $L(T)$ with the admissible lamination $L\left(S_{A}\right)$ which is the shift of bi-infinite admissible words in $A^{ \pm 1}$. In this setting a current if a shift-invariant, flip-invariant finite Borel measure on $L\left(S_{A}\right)$ Kap06, CHL08c].

For a current $\mu \in \mathbb{P} \operatorname{Curr}(T)$ and a word $w \in \mathbb{F}_{N}$, we denote by $\mu(w)=\mu\left(C_{A}(w)\right)$ the finite measure of the cylinder of $w$. As the cylinders generate the topology, the current $\mu$ is completly determined by the values $\mu(w)$, for $w \in \mathbb{F}_{N}$.

Proposition 2.2. Let $T$ be an $\mathbb{R}$-tree with a free minimal action of $\mathbb{F}_{N}$ by isometries with dense orbits. Let $\mu \in \mathbb{P} C \operatorname{urr}(T)$ be a current carried by the dual lamination of $T$. Then $\mu$ has no atomes.

Proof. Let $A$ be a basis of $\mathbb{F}_{N}$ and let $S_{A}=\left(K_{A}, A\right)$ be the associated system of isometries. Let $Z$ be a bi-infinite admissible word such that $\mu(Z)>0$. Then, as $\mu$ is shift-invariant, denoting by $\sigma$ the shift-map

$$
\mu\left(\left\{\sigma^{n} Z \mid n \in \mathbb{Z}\right\}\right)=\mu(Z) \cdot\left|\left\{\sigma^{n} Z \mid n \in \mathbb{Z}\right\}\right| \leq \mu\left(L\left(S_{A}\right)\right)<\infty .
$$

Thus the shift-orbit of $Z$ is finite and there exists $n>0$ such that $\sigma^{n} Z=Z$. Let $u$ be the prefix of the positive half $Z_{+}$of length $n$. We have $u^{-1} Z_{+}=Z_{+}$and using the equivariant map $\mathcal{Q}$

$$
\mathcal{Q}\left(Z_{+}\right)=u^{-1} \mathcal{Q}\left(Z_{+}\right) \text {. }
$$

But the action of $\mathbb{F}_{N}$ on $T$ is free, a contradiction.

2.5. Non-negative matrices. We recall here basic facts that are folklore in ergodic theory. The statements here are suitable in our context. In the analog context of interval exchange transformations we refer to the course of J.-C. Yoccoz [Yoc05] (in particular Corollary III.5 and the proof of Proposition IV.10).

For integers $d \geq 1,1 \leq i \leq d$ and $0 \leq j \leq d$ we consider the matrices $A_{d}^{i} \in M_{d \times(d+1)}\left(\mathbb{Z}_{\geq 0}\right)$ and $B_{d}^{i, j} \in M_{(d+1) \times d}\left(\mathbb{Z}_{\geq 0}\right):$

$$
A_{d}^{i}=\left(I_{d} \mid C_{d}^{i}\right) \text { et } B_{d}^{i, j}=\left(\begin{array}{c|c}
I_{j} & 0 \\
\hline & L_{d}^{i} \\
\hline 0 & I_{d-j}
\end{array}\right)
$$

where $C_{d}^{i}$ is the column vector of height $d$ with 0 coefficients except the $i$-th coefficient which is 1 and $L_{d}^{i}={ }^{t} C_{d}^{i}$ is the line vector of length $d$ with 0 coefficients except the $i$-th coefficient which is 1 .

Let $\left(d_{n}\right)$ be a sequence of positive integers and $\left(M_{n}\right)$ be a sequence of $d_{n} \times d_{n+1}$ integer non-negative matrices of the form $A_{d_{n}}^{i_{n}}$ or $B_{d_{n}}^{i_{n}, j_{n}}$. For such a sequence we denote by

$$
D=\liminf d_{n} .
$$

The positive cone of the sequence $\left(M_{n}\right)_{n \in \mathbb{N}}$ is the set

$$
C=\left\{\left(v_{n}\right)_{n \in \mathbb{N}} \mid \forall n \in \mathbb{N}, v_{n} \in \mathbb{R}_{\geq 0}^{d_{n}}, \text { and } v_{n}=M_{n} v_{n+1}\right\} .
$$

Lemma 2.3. The positive cone $C$ has projective dimension at most $D-1=\liminf d_{n}-1$.

Proof. By linearity, the dimension of $C$ is bounded above by $\lim \inf d_{n}$ and thus the projective dimension is bounded above by $\liminf d_{n}-1$.

We now state a Lemma that will be used to get a unique ergodicity criterion in Section 6 .

Lemma 2.4. Assume that there exists $L \geq 1$ and infinitely many $n$ such that

(1) $d_{n}=d_{n+L}=D$,

(2) the square matrix $M_{[n, n+L-1]}=M_{n} \cdots M_{n+L-1}$ has strictly positive entries.

Then, the projective positive cone defined by the sequence $\left(M_{n}\right)$ contains excatly one point.

Proof. The matrices $M_{[n, n+L-1]}$ are non-negative integer matrices. The entries of $M_{[n, n+L-1]}$ are bounded by some constant depending only on $L$. The matrices $M_{[n, n+L-1]}$ uniformly contract the Hilbert distance in the positive cone. Thus $C$ has zero diameter. 


\section{Levitt CASE}

3.1. Rips induction. Let $S=(F, A)$ be a system of isometries with graph $\Gamma$. Let $F^{\prime}$ be the set of elements of $F$ which belongs to the domains of at least two partial isometries in $A^{ \pm 1}$. The set $F^{\prime}$ is the union of the intersections of the domains of all possible pairs of distinct elements in $A^{ \pm 1}$ :

$$
F^{\prime}=\left\{P \mid \exists a \neq b \in A^{ \pm 1}, P \in \operatorname{dom}(a) \cap \operatorname{dom}(b)\right\}=\cup_{a \neq b \in A^{ \pm 1}} \operatorname{dom}(a) \cap \operatorname{dom}(b) .
$$

Thus $F^{\prime}$ is also a compact forest. Let $A^{\prime}$ be the set of all possible non-empty restrictions of elements of $A$ to pairs of connected components of $F^{\prime}$. The system of isometries $S^{\prime}=\left(F^{\prime}, A^{\prime}\right)$ is obtained from $S$ by $\mathbf{R i p s}$ induction.

Let $\Gamma^{\prime}$ be the graph of $S^{\prime}$ and let $\tau: \Gamma^{\prime} \rightarrow \Gamma$ map a vertex $K^{\prime}$ of $\Gamma^{\prime}$ (which is a connected component of $\left.F^{\prime}\right)$ to the connected component $\tau\left(K^{\prime}\right)=K$ of $F$ that contains $K^{\prime}$. Similarly, $\tau$ maps the edge $a^{\prime} \in A^{\prime \pm 1}$ to the edge $a \in A^{ \pm 1}$ of which $a^{\prime}$ is a restriction.

The system of isometries $S=(F, A)$ is reduced [CH14] if

(1) the graph $\Gamma$ is connected,

(2) it has independent generators,

(3) for each $P \in F$ there exists at least one infinite reduced admissible path $X$ such that $\mathcal{Q}(X)=P$ and,

(4) for each partial isometry $a \in A^{ \pm 1}$, and each extremal point $P$ in $\operatorname{dom}(a), P$ is in $F^{\prime}$.

We remark that if the system of isometries $S$ is reduced then the graph $\Gamma$ does not have vertices of valence 1.

We summarize our previous work in the following Proposition:

Proposition 3.1 ([CH14, Propostions 3.12, 3.13 and 5.6]). Let $S$ be a reduced system of isometries and $S^{\prime}$ be the system of isometries obtained by Rips induction. Then $S^{\prime}$ is reduced and the map $\tau: \Gamma^{\prime} \rightarrow \Gamma$ is a homotopy equivalence. Moreover, the map $\tau$ induces a one-to-one correspondance between bi-infinite admissible paths in $S$ and $S^{\prime}:$ For any bi-infinite admissible path $Z^{\prime} \in L\left(S^{\prime}\right)$, the bi-infinite path $\tau\left(Z^{\prime}\right)$ is reduced and admissible and, for any bi-infinite admissible path $Z$ in $\Gamma$ there exists a unique bi-infinite admissible path $Z^{\prime} \in L\left(S^{\prime}\right)$ such that $\tau\left(Z^{\prime}\right)=Z$. By abuse of notations we write

$$
L(S)=L\left(S^{\prime}\right)
$$

We now proceed to analyse cylinders of the lamination.

Proposition 3.2. Let $S=(F, A)$ be a reduced system of isometries with graph $\Gamma$. Let $S^{\prime}$ be obtained by Rips induction from $S$. Let $\Gamma^{\prime}$ be the graph of $S^{\prime}$ and $\tau: \Gamma^{\prime} \rightarrow \Gamma$ be the graph map.

Then, for each edge e of $\Gamma$

$$
C(\Gamma, e)=\biguplus_{e^{\prime}} C\left(\Gamma^{\prime}, e^{\prime}\right)
$$

where the disjoint union is taken over all edges $e^{\prime}$ of $\Gamma^{\prime}$ such that $\tau\left(e^{\prime}\right)=$ e. More generally, for any finite admissible path $w$ in $\Gamma$

$$
C(\Gamma, w)=\biguplus_{w^{\prime}} C\left(\Gamma^{\prime}, w^{\prime}\right) .
$$

where the disjoint union is taken over all finite reduced paths $w^{\prime}$ of $\Gamma^{\prime}$ such that $\tau\left(w^{\prime}\right)=w$.

Proof. First recall that the equalities in the Proposition are understood through the identifaction of $L(S)$ and $L\left(S^{\prime}\right)$ via the map $\tau$.

From Proposition 3.1, for each bi-infinite admissible path $Z \in C(\Gamma, w)$ there exists a unique bi-infinite admissible path $Z^{\prime} \in L\left(S^{\prime}\right)$ such that $\tau\left(Z^{\prime}\right)=Z$. Let $w^{\prime}$ be the prefix of $Z^{\prime}$ of length $|w|$, then $\tau\left(w^{\prime}\right)=w$. This proves that $Z^{\prime}$ is in $C\left(\Gamma^{\prime}, w^{\prime}\right)$ and that $w^{\prime}$ is unique. Conversely for any path $w^{\prime}$ of $\Gamma^{\prime}$ such that $\tau\left(w^{\prime}\right)=w$ and any bi-infinite reduced path $Z^{\prime} \in C\left(\Gamma^{\prime}, w^{\prime}\right), \tau\left(Z^{\prime}\right)$ is a bi-infinite reduced admissible path in $C(\Gamma, w)$. 
3.2. Analysis of the lamination. Let $T$ be an $\mathbb{R}$-tree with a free, minimal, action of the free group $\mathbb{F}_{N}$ with dense orbits. Let $A$ be a basis for $\mathbb{F}_{N}$, and let $S_{0}=S_{A}=\left(K_{A}, A\right)$ be the corresponding system of isometries. Recall that $S_{0}$ is reduced [CH14, Proposition 5.6]. We perform inductively Rips induction to get a sequence $S_{n}=\left(F_{n}, \Gamma_{n}\right)$ of reduced systems of isometries, together with maps $\tau_{n}: \Gamma_{n+1} \rightarrow \Gamma_{n}$. By Propositions 2.1 and 3.1, for each $n$ we have that $L(T)=L\left(S_{0}\right)=L\left(S_{n}\right)$.

For a vertex $v_{n}$ in $\Gamma_{n}$ we denote by $C\left(\Gamma_{n}, v_{n}\right)$ the set of bi-infinite admissible paths that goes through $v_{n}$ at index 0. From Proposition 3.1 we get that $\tau_{n-1}\left(C\left(\Gamma_{n}, v_{n}\right)\right) \subseteq C\left(\Gamma_{n-1}, \tau_{n-1}\left(v_{n}\right)\right)$. Again by abuse of notations we simply write $C\left(\Gamma_{n}, v_{n}\right) \subseteq C\left(\Gamma_{n-1}, \tau_{n-1}\left(v_{n}\right)\right)$. Recall also that $v_{n}$ is a connected component of the forest $F_{n}$ and that for each $Z \in C\left(\Gamma_{n}, v_{n}\right), \mathcal{Q}(Z)$ is a point in $v_{n}$.

Let now $\left(v_{n}\right)_{n \in \mathbb{N}}$ be a sequence such that for each $n \in \mathbb{N}, v_{n}$ is a vertex in $\Gamma_{n}$ and $\tau_{n}\left(v_{n+1}\right)=v_{n}$. The connected components $v_{n}$ of $F_{n}$ are nested and we denote by $v_{\infty} \subset K_{A}$ their intersection. We proved CH14] that the tree $T$ is of Levitt type if and only if for each such sequence $v_{\infty}$ consists of a single point. The cylinders $C\left(\Gamma_{n}, v_{n}\right)$ are also nested and their intersection is the set of bi-infinite admissible paths $Z$ in $\Gamma_{0}$ such that $\mathcal{Q}(Z) \in v_{\infty}$. As the action on $T$ is free, the map $\mathcal{Q}$ is finite-to-one [CH14, Corollary 5.4] and thus we proved

Proposition 3.3. Let $T$ be an $\mathbb{R}$-tree in $\partial c v_{N}$ with dense orbits and of Levitt type. Let $A$ be a basis of $\mathbb{F}_{N}$ and $\left(S_{n}\right)$ be the sequence of systems of isometries obtained from $S_{0}=S_{A}=\left(K_{A}, A\right)$ by Rips induction. Let $\left(v_{n}\right)$ be sequence of vertices of the graphs $\Gamma_{n}$ such that $\tau_{n}\left(v_{n+1}\right)=v_{n}$. Then, the nested intersection of the compact subtrees $v_{n}$ of $K_{A}$ is a singleton and the nested intersection of the cylinders $C\left(\Gamma_{n}, v_{n}\right)$ is finite.

3.3. Rips induction and currents. Let $T$ be an $\mathbb{R}$-tree with a free action of $\mathbb{F}_{N}$ by isometries with dense orbits. Let $A$ be a basis of $\mathbb{F}_{N}$ and $S_{0}=S_{A}=\left(K_{A}, A\right)$ be the associated system of isometries. Let $\left(S_{n}\right)_{n \in \mathbb{N}}$ be the sequence of systems of isometries obtained from $S_{0}$ by Rips induction. By Proposition 3.1 for each $n \in \mathbb{N}$, we have $L\left(S_{n}\right)=L(S)$. Let $\Gamma_{n}$ be the graph of $S_{n}$ and for each edge $e$ recall that $C\left(\Gamma_{n}, e\right)$ is the set of bi-infinite admissible paths in $\Gamma_{n}$ that goes through $e$ at index 0 .

For a current $\mu$ supported on the dual lamination $L(T)=L\left(S_{0}\right)=L\left(S_{n}\right)$, for each $n \in \mathbb{N}$, for each edge $e$ of $\Gamma_{n}$ we consider the finite number $\mu(e)=\mu\left(C\left(\Gamma_{n}, e\right)\right)$. If $e$ and $e^{\prime}$ are two consecutive edges of $\Gamma_{n}$ separated by a valence 2 vertex, any path through $e$ goes through $e^{\prime}$, and thus $\mu(e)=\mu\left(e^{\prime}\right)$. A generalized edge $\widehat{e}$ of $\Gamma_{n}$ is a maximal reduced path such that all inner vertices have valence exactly 2 . Recall that the maps $\tau_{n}$ are homotopy equivalences, that $\Gamma_{0}$ is the rose with $N$ petals and that the graphs $\Gamma_{n}$ are connected and do not have vertices of valence 1 . The graph $\Gamma_{n}$ has at most $3 N-3$ generalized edges. We denote by $\operatorname{GE}\left(\Gamma_{n}\right)$ the set of generalized edges of $\Gamma_{n}$. We pick-up arbitrarily an edge $e$ in each generalized edge $\widehat{e}$ in $\operatorname{GE}\left(\Gamma_{n}\right)$. As the system of isometries $S_{n}$ is reduced, there is a bi-infinite admissible path $Z$ going through each edge $e$ of $\Gamma_{n}$ and thus through each generalized edge $\widehat{e}$. By Propostion 3.1, $\tau_{0} \circ \cdots \circ \tau_{n-1}(Z)$ is a bi-infinite reduced path in $\Gamma_{0}$ and in particular its finite subpath $\tau_{0} \circ \cdots \circ \tau_{n-1}(\widehat{e})$ is reduced.

The incidence matrix $M_{n}$ of $\tau_{n}$ is the non-negative matrix such that for each pair of generalized edges $\widehat{e} \in \operatorname{GE}\left(\Gamma_{n}\right)$ and $\widehat{e}^{\prime} \in \operatorname{GE}\left(\Gamma_{n+1}\right)$, the coefficient $M_{n}\left(\widehat{e}, \widehat{e}^{\prime}\right)$ is the number of occurences of $e$ in the reduced finite path $\tau_{n}\left(\widehat{e}^{\prime}\right)$. We remark that this matrix depends on the choice of the edge $e$ in the generalized edge $\widehat{e}$.

To the current $\mu$ carried by $L(S)$, for each $n \in \mathbb{N}$, we associate the non-negative vector $\mu_{n}=(\mu(\widehat{e}))_{\widehat{e} \in \operatorname{GE}\left(\Gamma_{n}\right)}$.

Proposition 3.4. Let $T$ be an $\mathbb{R}$-tree with a free action of $\mathbb{F}_{N}$ by isometries with dense orbits. Let $A$ be a basis of $\mathbb{F}_{N}$ and $S_{0}=S_{A}=\left(K_{A}, A\right)$ be the associated system of isometries. Let $\left(S_{n}\right)_{n \in \mathbb{N}}$ be the sequence of systems of isometries obtained from $S_{0}$ by Rips induction. Let $\Gamma_{n}$ be the graph of $S_{n}$ and $G E\left(\Gamma_{n}\right)$ its set of generalized edge and, let $M_{n}$ be the incidence matrix of $\tau_{n}: \Gamma_{n+1} \rightarrow \Gamma_{n}$.

Then, for each current $\mu$ carried by $L(T)$

$$
\mu_{n}=M_{n} \mu_{n+1}
$$

where $\mu_{n}=\left(\mu\left(C\left(\Gamma_{n}, \widehat{e}\right)\right)\right)_{\widehat{e} \in G E\left(\Gamma_{n}\right)}$ is the non-negative vector associated to $\mu$ at step $n$.

Proof. From Proposition 3.2, we have

$$
\mu(\widehat{e})=\mu(e)=\sum_{e^{\prime}} \mu\left(e^{\prime}\right)=\sum_{e^{\prime}} \mu\left(\widehat{e}^{\prime}\right),
$$

where the sum is taken over all edges $e^{\prime}$ such that $\tau\left(e^{\prime}\right)=e$. Grouping together the generalized edges of $\Gamma^{\prime}$, proves the Proposition. 
We now use the fact that the tree is of Levitt type:

Proposition 3.5. With the above notations, let $\left(v_{n}\right)_{n \in \mathbb{N}}$ be a sequence such that for each $n, v_{n}$ is a vertex of $\Gamma_{n}$ and $\tau_{n}\left(v_{n+1}\right)=v_{n}$. Then the sequence $\left(\mu\left(C\left(\Gamma_{n}, v_{n}\right)\right)\right)_{n \in \mathbb{N}}$ is non-increasing and converges to 0 .

Proof. Recall from Section 3.2 that the cylinder $C\left(\Gamma_{n}, v_{n}\right)$ is the set of bi-infinite reduced admissible paths that goes through $v_{n}$ at index 0 . The cylinders $C\left(\Gamma_{n}, v_{n}\right)$ are nested and from Proposition 3.3 their intersection $C\left(v_{\infty}\right)$ is finite. By Proposition 2.2 the current $\mu$ has no atomes and $\mu\left(C\left(v_{\infty}\right)\right)$ is null, which proves the Proposition.

3.4. The simplex of currents. We are now ready to prove Theorem 1.1 in the case where the Rips induction completly decomposes the tree.

Theorem 3.6. Let $T$ be an $\mathbb{R}$-tree with a minimal free action of $\mathbb{F}_{N}$ by isometries with dense orbits. Assume that $T$ is a tree of Levitt type.

Then the simplex $\mathbb{P} C \operatorname{cur}(T)$ of currents carried by the dual lamination $L(T)$ has dimension at most $3 N-4$ (and projective dimension at most $3 N-5$ ).

Proof. Let $A$ be a basis of $\mathbb{F}_{N}$ and $S_{0}=S_{A}=\left(K_{A}, A\right)$ be the associated system of isometries. We perform the Rips induction to get a sequence of systems of isometries $S_{n}$ together with maps $\tau_{n}: \Gamma_{n+1} \rightarrow \Gamma_{n}$.

For each $\mu \in \mathbb{P C u r r}(T)$, we consider the sequence of vectors $\left(\mu_{n}\right)_{n \in \mathbb{N}}$, where $\mu_{n}=\left(\mu_{n}(\widehat{e})\right)_{\widehat{e} \in \operatorname{GE}\left(\Gamma_{n}\right)}$. We denote by $C=\left\{\left(\mu_{n}\right)_{n \in \mathbb{N}} \mid \mu \in \mathbb{P C u r r}(T)\right\}$ the positive cone spanned by these vectors. For each $n$, let $M_{n}$ be the incidence matrix of the map $\tau_{n}$. From Proposition 3.4. $C$ is the cone associated to the sequence of matrices $\left(M_{n}\right)$ as in Section 2.5.

The incidence matrices $M_{n}$ are products of matrices of the form $A_{d}^{i}$ and $B_{d}^{i, j}$ (see Section 2.5). Indeed, the matrix $A_{d}^{i}$ is that of splitting the $i$-th edge of $\Gamma_{n}$ into two edges and the matrix $B_{d}^{i, j}$ is that of replacing the reduced path made of the the $i$-th and $j$-th edges of $\Gamma_{n+1}$ when they form a generalized edge. Edges splitted in the Rips induction are incident to branch vertices of $\Gamma_{n}$ and the number of resulting splitted edges is at most the valence of that branch vertex. Moreover, these splittings creates as many vertices as the number of new edges. Thus, both the numbers of matrices $A_{d}^{i}$ and $B_{d}^{j}$ in each $M_{n}$ is bounded by $2 N-2$. Because $T$ is of Levitt type, the Rips machine goes for ever and the number of edges of $\Gamma_{n}$ goes to infinity.

From Lemma 2.3, the projective dimension of $C$ is at most $D-2$ where $D$ is the inferior limit of the number of generalized edges in $\Gamma_{n}$. As $\Gamma_{n}$ is homotopic to the rose with $N$-petals, the number of generalized edges is bounded above by $3 N-3$. The Rips induction applied to a graph $\Gamma_{n}$ with $3 N-3$ generalized edges eventually produces a graph with strictly less than $3 N-3$ generalized edges. Thus we get that $D<3 N-3$ and the projective dimension of $C$ is at most $3 N-5$.

Recall from Section 2.4 that for a finite reduced word $w$ in $A^{ \pm 1}$ the cylinder $C_{A}(w) \subseteq \partial^{2} \mathbb{F}_{N}$ is the clopen set of bi-infinite reduced words in $A^{ \pm 1}$ that reads $w$ at index 0 . The set of translates of all such cylinders is a sub-basis of open sets of the shift of bi-infinite reduced words in $A^{ \pm 1}$. Thus a current is completely determined by the measures of these cylinders.

Recall that a a generalized edge $\widehat{e}$ in $\Gamma_{n}$ is mapped by $\tau_{0} \circ \cdots \tau_{n-1}$ to a finite reduced admissible path in $\Gamma_{0}$ and that $\Gamma_{0}$ is the rose with $N$ petals labelled by $A$.

We denote by $\langle\widehat{e} \mid w\rangle$ the number of occurences of the word $w$ in the reduced word $\tau_{0} \circ \cdots \tau_{n-1}(\widehat{e})$. We claim that for a current $\mu \in \mathbb{P} \operatorname{Curr}(T)$,

$$
\mu(w)=\lim _{n \rightarrow+\infty} \sum_{\widehat{e} \in \operatorname{GE}\left(\Gamma_{n}\right)}\langle\widehat{e} \mid w\rangle \mu(\widehat{e}) .
$$

This formula proves that $\mu$ is completely determined by the image of $\mu$ in $C$ and thus proves the Proposition. We now prove the above formula.

Using Proposition 3.1, we get

$$
C\left(\Gamma_{0}, w\right)=\biguplus_{w^{\prime}} C\left(\Gamma_{n}, w^{\prime}\right)
$$

where the disjoint union is taken over all reduced paths $w^{\prime}$ in $\Gamma_{n}$ with label $w$. Passing to the current we get

$$
\mu(w)=\sum_{w^{\prime}} \mu\left(C\left(\Gamma_{n}, w^{\prime}\right)\right) .
$$


If $w^{\prime}$ is a subpath of a generalized edge $\widehat{e}$ of $\Gamma_{n}$, then all bi-infinite reduced paths through $w^{\prime}$ goes through $\widehat{e}$ and thus $\mu\left(C\left(\Gamma_{n}, w^{\prime}\right)\right)=\mu(\widehat{e})$. Taking into account only these occurences of the label $w$ inside generalized edges, we get the inequality

$$
\mu(w) \geq \sum_{\widehat{e} \in \operatorname{GE}\left(\Gamma_{n}\right)}\langle\widehat{e} \mid w\rangle \mu(\widehat{e}) .
$$

The difference between the two terms above is exactly the measure of the cylinders of the occurences of the label $w$ that are not inside a generalized edge. Those occurences cross a point of valence at least 3 . For such a vertex $v$ in $\Gamma_{n}$ of valence at least 3 , we denote by $\langle v \mid w\rangle$ the number of reduced paths in $\Gamma_{n}$ with label $w$ that pass through $v$. We remark that $\langle v \mid w\rangle$ is bounded above by $(|w|-1)(2 N-1)^{|w|}$, where $|w|-1$ is the number of vertices inside a path of length $|w|$ and $2 N$ is the maximal valence of a vertex in $\Gamma_{n}$. (Note that this bound is verly loose but sufficient for our proof). We also use the notation $\mu(v)=\mu\left(C\left(\Gamma_{n}, v\right)\right)$. The difference of the two terms in the above inequality is bounded by

$$
0 \leq \mu(w)-\sum_{\widehat{e} \in \operatorname{GE}\left(\Gamma_{n}\right)}\langle\widehat{e} \mid w\rangle \mu(e) \leq \sum_{v}\langle v, w\rangle \mu(v) \leq(|w|-1)(2 N-1)^{|w|} \sum_{v} \mu(v)
$$

where $v$ ranges over all branch-points of $\Gamma_{n}$. Now $\Gamma_{n}$ is homotopy equivalent to $\Gamma_{0}$ and does not have vertices of valence 1, thus the number of branch-points in $\Gamma_{n}$ is bounded above by $2 N-2$. We get

$$
0 \leq \mu(w)-\sum_{\widehat{e} \in \operatorname{GE}\left(\Gamma_{n}\right)}\langle\widehat{e} \mid w\rangle \mu(e) \leq(|w|-1)(2 N-1)^{|w|}(2 N-2) \max _{v} \mu(v)
$$

By Proposition 3.5 the last factor goes to 0 when $n$ goes to infinity which concludes the proof.

\section{Splitting induction}

There are trees in the boundary of Outer space where the Rips induction is unuseful: the trees of surface type [CH14]. For these trees (and in general) we define another kind of induction, which we call splitting induction. It is a little more involved to use it for analysing the dual lamination as we are going to miss finitely many leaves. But this gives us the same results for currents when they have no atomes.

4.1. Splitting. This Section recalls the definitions and results of our previous work with P. Reynolds CHR11, Sections 4.2 and 4.3$]$.

Let $S=(F, A)$ be a reduced system of isometries. A point $P \in F$ is in the interior of a tree $K \subseteq F$ if $P$ is in the interior of a segment contained in $K$. A point $P$ is extremal in a tree $K$ if it is not in the interior.

Let $x$ be an interior point of a connected component $K_{x}$ of $F$. Let $\pi_{0}\left(K_{x} \backslash\{x\}\right)=L \biguplus R$ be a partition of the set of directions at $x$ in two non-empty subsets. Then $(x, L, R)$ is a splitting partition of the system of isometries $S=(F, A)$ if

(1) there exists exactly one partial isometry $a_{0} \in A^{ \pm 1}$ defined at $x$ whose domain $\operatorname{dom}\left(a_{0}\right)$ meets both sets of directions $L$ and $R$ and,

(2) for each direction $d \in L \cup R$ at $x$, there is at least another partial isometry $a \in A^{ \pm 1} \backslash\left\{a_{0}\right\}$ defined at $d: x \in \operatorname{dom}(a)$ and $\operatorname{dom}(a) \cap d \neq \emptyset$.

For a splitting partition $(x, L, R)$, let $F^{\prime}$ be the forest obtained by splitting $K_{x}$ into two disjoint compact trees $\bar{L}$ and $\bar{R}$ :

$$
F^{\prime}=\left(F \backslash K_{x}\right) \biguplus(L \cup\{x\}) \biguplus(R \cup\{x\}) .
$$

We insist that there are two distinct copies of $x$ in $F^{\prime}$, which we denote by $x_{L}$ and $x_{R}$.

We also split the partial isometries in $A$. The partial isometry $a_{0}$ is replaced by its two restrictions:

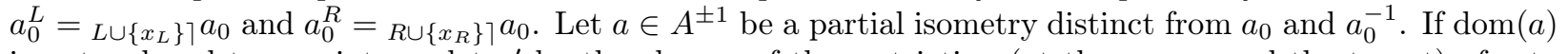
is not reduced to a point, we let $a^{\prime}$ be the closure of the restriction (at the source and the target) of $a$ to $F \backslash\{x\}$. For instance, if $x$ is in the domain of $a$ then by definition of a splitting point, $x$ is extremal in $\operatorname{dom}(a)$ and $\operatorname{dom}(a)$ meets either $L$ or $R$, say $L$. In this case $\operatorname{dom}\left(a^{\prime}\right)=\left\{x_{L}\right\} \cup(\operatorname{dom}(a) \cap L)$. Finally, if 
$\operatorname{dom}(a)$ is a singleton $\{y\}$ and $y \cdot a=y^{\prime}$ we define $a^{\prime}$ arbitrarily by letting

$$
\left\{\begin{array}{l}
y \cdot a^{\prime}=y^{\prime} \text { if } x \notin\left\{y, y^{\prime}\right\} \\
x_{L} \cdot a^{\prime}=x_{L} \text { if } y=y^{\prime}=x \\
x_{L} \cdot a^{\prime}=y^{\prime} \text { if } y=x \text { and } y^{\prime} \neq x \\
y \cdot a^{\prime}=x_{L} \text { if } y \neq x \text { and } y^{\prime}=x .
\end{array}\right.
$$

Considering the graphs $\Gamma$ and $\Gamma^{\prime}$ of the systems of isometries $S$ and $S^{\prime}$, as above there is a natural map $\tau: \Gamma^{\prime} \rightarrow \Gamma$ which is one-to-one, except that it maps both vertices $\bar{L}$ and $\bar{R}$ of $\Gamma^{\prime}$ to the vertex $K_{x}$ of $\Gamma$ and, except that it maps both edges $a_{0}^{L}$ and $a_{0}^{R}$ of $\Gamma^{\prime}$ to the edge $a_{0}$ of $\Gamma$. Indeed the map $\tau$ is a folding.

The splitting induction affects the admissible paths that contain the subpaths $a_{0}^{R^{-1}} a_{0}^{L}$ or $a_{0}^{L^{-1}} a_{0}^{R}$. We thus restrict to a sublamination. A finite admissible path $u$ in $\Gamma$ is regular if $\operatorname{dom}(u)$ contains strictly more than one point. The regular lamination $L^{\prime}(S)$ of a system of isometries $S$ is the set of bi-infinite admissible paths $Z$ in $\Gamma$ such that all finite subpaths $u$ of $Z$ are regular. This regular lamination is also the derived set $L^{\prime}(S)$ of non-isolated leaves in $L(S)$ :

Proposition 4.1 ([CHR11]). Let $T$ be an $\mathbb{R}$-tree with a free minimal action of $\mathbb{F}_{N}$ by isometries with dense orbits. Let $A$ be a basis of $\mathbb{F}_{N}$ and $S_{A}=\left(K_{A}, A\right)$ be the associated system of isometries. Recall that the dual lamination $L(T)$ of $T$ is equal to the admissible lamination of $S_{A}$.

Then, the regular lamination $L^{\prime}\left(S_{A}\right)$ is the derived space of $L\left(S_{A}\right)$.

We summarize our previous work with P. Reynolds in the following Proposition:

Proposition 4.2 ([CHR11, Lemma 4.8 and 4.9]). Let $S$ be a reduced system of isometries and let $S^{\prime}$ be a system of isometries obtained by splitting. Then $S^{\prime}$ is reduced and the map $\tau: \Gamma^{\prime} \rightarrow \Gamma$ is a homotopy equivalence.

For any bi-infinite reduced regular path $Z^{\prime} \in L\left(S^{\prime}\right)$ in $\Gamma^{\prime}$, the bi-infinite path $\tau\left(Z^{\prime}\right)$ is reduced, admissible and regular. For any bi-infinite reduced regular path $Z \in L(S)$ in $\Gamma$, there exists a unique bi-infinite reduced regular path $Z^{\prime} \in L\left(S^{\prime}\right)$ such that $\tau\left(Z^{\prime}\right)=Z$.

Through the map $\tau$, we identify the regular laminations:

$$
L^{\prime}(S)=L^{\prime}\left(S^{\prime}\right) .
$$

We now write the effect of splitting on the cylinders of the lamination of $S$. As in Section 3 for a finite reduced path $u$ of $\Gamma$ ( $u$ can be a vertex or an edge), the cylinder $C(\Gamma, u)$ is the set of bi-infinite admissible paths that goes through $u$ at index 0 . But here we rather consider the regular cylinder $C^{\prime}(\Gamma, u)$ : the set of bi-infinite reduced admissible regular paths that goes through $u$ at index 0. Exactly as in Proposition 3.2 we have:

Proposition 4.3. Let $S=(F, A)$ be a reduced system of isometries with graph $\Gamma$. Let $S^{\prime}$ be obtained by splitting induction from $S$. Let $\Gamma^{\prime}$ be the graph of $S^{\prime}$ and $\tau: \Gamma^{\prime} \rightarrow \Gamma$ be the graph map. For each edge e of $\Gamma$ :

$$
C^{\prime}(\Gamma, e)=\biguplus_{e^{\prime}} C^{\prime}\left(\Gamma^{\prime}, e^{\prime}\right)
$$

where the disjoint union is taken over all edges $e^{\prime}$ of $\Gamma^{\prime}$ such that $\tau\left(e^{\prime}\right)=e$. More generally, for every finite regular path $u$ in $\Gamma$

$$
C^{\prime}(\Gamma, u)=\biguplus_{u^{\prime}} C^{\prime}\left(\Gamma^{\prime}, u^{\prime}\right)
$$

where the disjoint union is taken over all finite reduced paths $u^{\prime}$ of $\Gamma^{\prime}$ such that $\tau\left(u^{\prime}\right)=u$.

4.2. Existence of splittings. To use the splitting induction to analyse laminations and currents we first need to prove that we can perform it: there exists splitting partitions. From our previous work with P. Reynolds [CHR11] we know that this is the case, at least when we cannot perform Rips induction. A system of isometries $S=(F, A)$ is of surface type if any point $x$ of $F$ belongs to the domains of at least two partial isometries $a \neq b \in A^{ \pm 1}$ (equivalently Rips induction does nothing to $S$ ).

Proposition 4.4 (CHR11, Proposition 4.11]). Let $S=(F, A)$ be a reduced system of isometries of surface type, then there exists a splitting partition for $S$. 


\section{UNFOLDING}

5.1. Unfolding induction and currents. Let $T$ be an $\mathbb{R}$-tree with a free minimal action of $\mathbb{F}_{N}$ by isometries with dense orbits. Let $L(T)$ be the dual lamination of $T$. By Proposition 2.2 , no leaf of $L(T)$ is periodic and, any current $\mu \in \mathbb{P C u r r}(T)$ carried by $L(T)$ has no atomes. By definition of the derived space we get:

Proposition 5.1. Let $T$ be an $\mathbb{R}$-tree with a free minimal action of $\mathbb{F}_{N}$ by isometries with dense orbits. Then every current $\mu \in \mathbb{P} C$ Curr $(T)$ carried by the dual lamination $L(T)$ is carried by the regular lamination $L^{\prime}(T)$.

Let $A$ be a basis of $\mathbb{F}_{N}$ and $S_{A}=\left(K_{A}, A\right)$ the associated system of isometries. Let $S_{n}=\left(F_{n}, A_{n}\right)$ be a sequence of systems of isometries obtained from $S_{0}=S_{A}$ by unfolding induction: at each step $n$ either Rips or splitting induction is performed. Let $\tau_{n}: \Gamma_{n+1} \rightarrow \Gamma_{n}$ be the map between the graphs. As $S_{0}$ is reduced, $\tau_{n}$ is a homotopy equivalence, $\Gamma_{n}$ is connected and does not have vertices of valence 1 . The graph $\Gamma_{n}$ has at most $3 N-3$ generalized edges in $\mathrm{GE}\left(\Gamma_{n}\right)$.

For each generalized edge $\widehat{e}^{\prime} \in \operatorname{GE}\left(\Gamma_{n+1}\right)$ and each edge $e$ in $\Gamma_{n}$ we consider $\left\langle\widehat{e}^{\prime}, e\right\rangle$ the number of occurences of $e$ (without taking into account orientation) in the finite path $\tau(\widehat{e})$. The incidence matrix $M_{n}$ of the map $\tau_{n}$ has entry $\left\langle\widehat{e}^{\prime}, e\right\rangle$ for each pair $\left(\widehat{e}, \widehat{e}^{\prime}\right) \in \operatorname{GE}\left(\Gamma_{n}\right) \times \operatorname{GE}\left(\Gamma_{n+1}\right)$. Again, we remark that the incidence matrix depends on the choice of the edge $e$ in the generalized edge $\widehat{e}$.

For a generalized edge $\widehat{e}$ of $\Gamma_{n}$ we consider the non-negative number $\mu\left(C^{\prime}\left(\Gamma_{n}, \widehat{e}\right)\right)$, where $C^{\prime}\left(\Gamma_{n}, \widehat{e}\right)$ is the cylinder of regular bi-infinite admissible paths in $\Gamma_{n}$ that passes through $\widehat{e}$ at index 0 . We consider the non-negative vector $\mu_{n}=\left(\mu\left(C^{\prime}\left(\Gamma_{n}, \widehat{e}\right)\right)\right)_{\widehat{e} \in \mathrm{GE}\left(\Gamma_{n}\right)}$.

Proposition 5.2. Let $T$ be an $\mathbb{R}$-tree with a free action of $\mathbb{F}_{N}$ by isometries with dense orbits. Let $A$ be a basis of $\mathbb{F}_{N}$ and $S_{0}=S_{A}=\left(K_{A}, A\right)$ be the associated system of isometries. Let $\left(S_{n}\right)_{n \in \mathbb{N}}$ be a sequence of systems of isometries obtained from $S_{0}$ by unfolding induction. Let $\Gamma_{n}$ be the graph of $S_{n}$ and $G E\left(\Gamma_{n}\right)$ its set of generalized edge and, let $M_{n}$ be the incidence matrix of $\tau_{n}: \Gamma_{n+1} \rightarrow \Gamma_{n}$.

Then, for each current $\mu$ carried by $L(T)$

$$
\mu_{n}=M_{n} \mu_{n+1}
$$

where $\mu_{n}=\left(\mu\left(C^{\prime}\left(\Gamma_{n}, \widehat{e}\right)\right)\right)_{\widehat{e} \in G E\left(\Gamma_{n}\right)}$ is the non-negative vector associated to $\mu$ at step $n$.

Proof. The proof is the same as the proof of Proposition 3.4, using Proposition 4.3 if we use splitting induction at step $n$.

We can now prove Theorem 1.1 when the induction completly analyses the lamination.

Theorem 5.3. Let $T$ be an $\mathbb{R}$-tree with a minimal, free, action of $\mathbb{F}_{N}$ by isometries with dense orbits. Let $A$ be a basis of $\mathbb{F}_{N}$ and let $S_{A}=\left(K_{A}, A\right)$ be the associated system of isometries. Let $S_{n}=\left(F_{n}, A_{n}\right)$ be a sequence of systems of isometries obtained from $S_{0}=S_{A}$ by unfolding induction.

Assume that each nested intersection of connected components $v_{n}$ of $F_{n}$ is a singleton.

Then, the simplex of currents $\mathbb{P} C u r r(T)$ carried by the dual lamination $L(T)$ has dimension at most $3 N-4$ (and projective dimension at most $3 N-5$ ).

Proof. Again the proof is the same as the proof of Theorem 3.6, using the regular lamination instead of the lamination and Proposition [5.2.

5.2. Decomposable case. The hypotesis of Theorem 5.3 are not always satisfied: there exist $\mathbb{R}$-trees with no induction sequence that completly analyses the lamination. Those trees are decomposable in the sense of V. Guirardel Gui08]. In this case however, the induction procedure ends up with a subtree with an action of a subgroup of $\mathbb{F}_{N}$ with rank strictly smaller than $N$. And this allows us to conclude the proof of Theorem 1.1 by induction on $N$.

Let $T$ be an $\mathbb{R}$-tree with a minimal free action of $\mathbb{F}_{N}$ with dense orbits. Let $A$ be a basis of $\mathbb{F}_{N}$ and let $S_{A}=\left(K_{A}, A\right)$ be the associated system of isometries. Let $S_{n}=\left(F_{n}, A_{n}\right)$ be an infinite sequence of systems of isometries obtained from $S_{0}=S_{A}$ by unfolding induction. By Proposition 4.4 this is always possible. Let $\tau_{n}: \Gamma_{n+1} \rightarrow \Gamma_{n}$ be the map between the graphs. As $S_{n}$ is reduced, $\Gamma_{n}$ is connected, does not have vertices of valence 1 and $\tau_{n}$ is a homotopy equivalence. The number of edges of $\Gamma_{n}$ is strictly increasing with $n$. The inverse limit of $\left(\Gamma_{n}, \tau_{n}\right)$ is an infinite graph $\widehat{\Gamma}$. Vertices of $\widehat{\Gamma}$ are sequences $\left(v_{n}\right)_{n \in \mathbb{N}}$ such that $v_{n}$ is a vertex 
of $\Gamma_{n}$ and $\tau_{n}\left(v_{n+1}\right)=v_{n}$, thus $\left(v_{n}\right)_{n \in \mathbb{N}}$ is a sequence of nested compact subtrees of $K_{A}$. Edges of $\widehat{\Gamma}$ are sequences $\left(a_{n}\right)_{n \in \mathbb{N}}$ such that $a_{n}$ is an edge of $\Gamma_{n}$ and $\tau_{n}\left(a_{n+1}\right)=a_{n}$, thus $a_{n+1}$ is a restriction of the partial isometry $a_{n}$.

By our previous work [CH14, the index of $\widehat{\Gamma}$ is finite and thus it contains a finite core graph $\Gamma_{\infty}$ : the union of all reduced loops in $\widehat{\Gamma}$. We remark that $\Gamma_{\infty}$ can be empty and that it can fail to be connected.

First case: $\Gamma_{\infty}$ is empty. Equivalently, each connected component of $\widehat{\Gamma}$ is a tree. Moreover, as the index of $\widehat{\Gamma}$ is finite, each connected component of $\widehat{\Gamma}$ has a finite number of ends. Let $\left(v_{n}\right)$ be a vertex of $\widehat{\Gamma}$, this is a sequence of vertices of $\Gamma_{n}$ which are nested connected components of the forest $F_{n}$. As before, we denote by $v_{\infty}$ the nested intersection of $\left(v_{n}\right)$. There are finitely many infinite reduced paths in $\widehat{\Gamma}$ starting from $v_{\infty}$. For each $x \in v_{\infty}$ there exists an infinite path in $\widehat{\Gamma}$ starting at $\left(v_{n}\right)$ which reads an admissible reduced regular word $X$ such that $\mathcal{Q}(X)=x$. We get that $v_{\infty}$ is finite and thus a singleton. Theorem 5.3 applies in this case.

Second case: $\Gamma_{\infty}$ is non-empty. As $\Gamma_{\infty}$ is finite, there exists $s \in \mathbb{N}$ such that for all $n \geq s, \Gamma_{\infty}$ is a subgraph of $\Gamma_{n}$ and the map $\tau_{n}$ restricts to the identity on $\Gamma_{\infty}$. We denote by $\Gamma_{\infty}^{1}, \ldots, \Gamma_{\infty}^{r}$ the connected components of $\Gamma_{\infty}$ in this case $r \geq 1$. For each $i=1, \ldots, r$, let $F_{\infty}^{i}$ be the compact forest whose connected components are the nested intersections of the vertices of $\Gamma_{\infty}^{i}$, and let $A_{\infty}^{i}$ be the partial isometries of $F_{\infty}^{i}$ which are the nested intersections of edges of $\Gamma_{\infty}^{i}$.

Lemma 5.4. The system of isometries $S_{\infty}^{i}=\left(F_{\infty}^{i}, A_{\infty}^{i}\right)$ has graph $\Gamma_{\infty}^{i}$ and, is reduced.

Let $N_{i}$ be the rank of the free group $\pi_{1}\left(\Gamma_{\infty}^{i}\right)$. Let $T_{i}$ be the $\mathbb{R}$-tree associated to $S_{\infty}^{i}$. The action of $F_{N_{i}}$ on $T_{i}$ is free, minimal by isometries and with dense orbits.

For any finite admissible non-singular subpath $u$ in $\Gamma_{\infty}^{i}$, we consider the regular cylinder $C^{\prime}\left(\Gamma_{\infty}^{i}, u\right)$ of bi-infinite admissible regular paths that passes through $u$ at index 0 . The translates of such cylinders form a basis of open sets of the regular dual lamination $L^{\prime}\left(T_{i}\right)$. We insist that we consider $L^{\prime}\left(T_{i}\right)$ as a lamination with respect to $F_{N_{i}}=\pi_{1}\left(\Gamma_{\infty}^{i}\right)$ (and not with respect to the original $\mathbb{F}_{N}$ ). Any current $\mu \in \mathbb{P C u r r}(T)$ carried by the dual lamination $L(T)$ induces a current $\mu^{i} \in \mathbb{P C u r r}\left(T_{i}\right)$ by letting

$$
\mu^{i}\left(C\left(\Gamma_{\infty}^{i}, u\right)\right)=\mu\left(C\left(\Gamma_{\infty}^{i}, u\right)\right) .
$$

For each $n>s$, as the homotopy equivalence $\tau_{n}$ fixes the subgraph $\Gamma_{\infty}$ the incidence matrix $M_{n}$ is reducible and we denote by $M_{n}^{\mathrm{GE}}$ its submatrix corresponding to generalized edges in $\operatorname{GE}\left(\Gamma_{n} \backslash \Gamma_{\infty}\right)$. Let $C$ be the positive cone of non-negative vectors $\left(\left(\mu_{n}(\widehat{e})\right)_{\widehat{e} \in \operatorname{GE}\left(\Gamma_{n} \backslash \Gamma_{\infty}\right)}\right)_{n \geq s}$.

Lemma 5.5. The map

$$
\begin{aligned}
\mathbb{P} C u r r(T) & \rightarrow \mathbb{P} \operatorname{Curr}\left(T_{1}\right) \times \cdots \times \mathbb{P} C u r r\left(T_{r}\right) \times C \\
\mu & \mapsto\left(\mu^{1}, \ldots, \mu^{r},\left(\left(\mu_{n}(\widehat{e})\right)_{\left.\left.\widehat{e} \in G E\left(\Gamma_{n} \backslash \Gamma_{\infty}\right)\right)_{n \geq s}\right)}\right.\right.
\end{aligned}
$$

is injective.

Proof. Indeed for any finite reduced word $w$ in $A^{ \pm 1}$, we claim that

$$
\mu(w)=\mu\left(C\left(\Gamma_{0}, w\right)\right)=\sum_{i=1}^{i=r} \sum_{w^{\prime} \text { occurence of } w \text { in } \Gamma_{\infty}^{i}} \mu^{i}\left(w^{\prime}\right)+\lim _{n \rightarrow \infty} \sum_{\widehat{e} \in \operatorname{GE}\left(\Gamma_{n} \backslash \Gamma_{\infty}\right)}\langle w, \widehat{e}\rangle \mu(\widehat{e}) .
$$

As in the proof of Theorem 3.6. Propositions 3.1 and 4.2 prove that for each $n$ :

$$
\mu(w) \geq \sum_{i=1}^{i=r} \sum_{w^{\prime} \text { occurence of } w \text { in } \Gamma_{\infty}^{i}} \mu^{i}\left(w^{\prime}\right)+\sum_{\widehat{e} \in \operatorname{GE}\left(\Gamma_{n} \backslash \Gamma_{\infty}\right)}\langle w, \widehat{e}\rangle \mu(\widehat{e}) .
$$

and that the difference is given by

$$
\Delta_{n}=\sum_{w^{\prime}} \mu\left(C^{\prime}\left(\Gamma_{n}, w^{\prime}\right)\right)
$$

where the sum is taken over all occurences of $w$ as a label of a path $w^{\prime}$ in $\Gamma_{n}$ that contains both vertices of $\Gamma_{\infty}$ and edges of $\Gamma_{n} \backslash \Gamma_{\infty}$. By definition of $\Gamma_{\infty}$, all vertices of $\Gamma_{\infty}$ have valence at least 2 and thus those occurences of $w$ passes over one of the branch points of $\Gamma_{n}$. Therefore the number of such occurences is uniformly bounded (by a constant which depends only on $N$ ). Hence, there are finitely many occurences of 
$w$ in the inverse limit $\widehat{\Gamma}$ which contains both vertices of $\Gamma_{\infty}$ and edges out of $\Gamma_{\infty}$. The vertices out of the core graph of $\widehat{\Gamma}$ corresponds to singletons in $K_{A}$ and as the action of $\mathbb{F}_{N}$ on $T$ is free there are only finitely many bi-infinite admissible regular paths $Z$ in $\widehat{\Gamma}$ that passes through these occurences of $w$ at index 0 .

We thus get that $\lim _{n \rightarrow \infty} \Delta_{n}=0$

By Propostion 5.2 and by definition of the cone $C$, for all $\left(v_{n}\right)_{n \geq s} \in C$ and for $n \geq s$, we have $v_{n}=$ $M_{n}^{\mathrm{GE}} v_{n+1}$. Thus, the cone $C$ has finite dimension at most the number of generalized edges in $\Gamma_{n} \backslash \Gamma_{\infty}$ minus one. By induction on the rank of the free group, $\mathbb{P C u r r}\left(T_{i}\right)$ has dimension at most $3 N_{i}-5$. Let $\Gamma_{n}^{\mathrm{GE}}$ be the graph obtained from $\Gamma_{n}$ by replacing generalized edges by edges (so that $\Gamma_{n}^{\mathrm{GE}}$ has no vertex of valence 2). Contracting the image of each component $\Gamma_{\infty}^{i}$ in $\Gamma_{n}^{\mathrm{GE}}$ to a vertex, we get a connected graph $G_{n}$. By construction $G_{n}$ has no vertex of valence 1 , at most $r$ vertices of valence 2 and $\left|\operatorname{GE}\left(\Gamma_{n} \backslash \Gamma_{\infty}\right)\right|$ edges. Moreover $G_{n}$ has Euler characteristic $1-N+N_{1}+\cdots+N_{r}$. Using that the number of edges of a connected graph without vertex of valence 1 is bounded above by three times the Euler characteristic plus the number of vertices of valence 2 , we get

$$
\left|\mathrm{GE}\left(\Gamma_{n} \backslash \Gamma_{\infty}\right)\right| \leq 3\left(N-N_{1}-\cdots-N_{r}\right)-3+r
$$

and thus

$$
\left(3 N_{1}-4\right)+\cdots+\left(3 N_{r}-4\right)+\left|\operatorname{GE}\left(\Gamma_{n} \backslash \Gamma_{\infty}\right)\right|-1 \leq 3 N-4-3 r
$$

which concludes the proof of Theorem 1.1 since $r \geq 1$.

\section{UNIQUE ERGODICITY}

Recall that an $\mathbb{R}$-tree in $\partial \mathrm{CV}_{N}$ is uniquely ergodic if it is dual to a unique projective current. There are known examples of uniquely ergodic trees: As the transition matrix of a train-track of an iwip outer automorphisms is a primitive integer matrix (up to passing to a power), Perron-Frobenius Theorem implies that the attracting tree in $\partial \mathrm{CV}_{N}$ of a non-geometric iwip outer automorphims is uniquely ergodic - see for instance CHL08c].

In this Section we give a more general concrete criterion of unique ergodicity.

Let $T$ be an $\mathbb{R}$-tree in $\partial \mathrm{CV}_{N}$ with dense orbits. Let $A$ be a basis of $\mathbb{F}_{N}$, and $\left(S_{n}\right)$ a sequence of systems of isometries obtained from $S_{0}=S_{A}=\left(K_{A}, A\right)$ by unfolding induction. As in Section 3.2 and [5.1, let $\left(\Gamma_{n}\right)$ be the sequence of associated graphs and $\tau_{n}: \Gamma_{n+1} \rightarrow \Gamma_{n}$ the homotopy equivalences. We denote by $D$ the inferior limit of the number $d_{n}$ of generalized edges of $\Gamma_{n}$ :

$$
D=\liminf d_{n} .
$$

We say that all edges are fully splitted between steps $n$ and $n+L$ if the image of each generalized edge $\widehat{e}$ of $\Gamma_{n+L}$ in $\Gamma_{n}$ is an edge-path that covers $\Gamma_{n}$. This is equivalent to saying that the incidence matrix (for generalized edges) of $\tau_{n} \circ \cdots \circ \tau_{n+L-1}: \Gamma_{n+L} \rightarrow \Gamma_{n}$ has strictly positive entries.

Theorem 6.1. Assume that there exists $L$ and infinitely many $n$ such that the number of generalized edges of $\Gamma_{n}$ is $D$ and between steps $n$ and $n+L$ all edges are fully splitted.

Then, $T$ is uniquely ergodic.

Proof. Indeed with the hypothesis above the matrices $M_{n} \cdots M_{n+L-1}$ are square matrices with strictly positive and bounded coefficients. Thus we can use Lemma 2.4.

\section{REFERENCES}

[BF95] Mladen Bestvina and Mark Feighn. Stable actions of groups on real trees. Invent. Math., 121(2):287-321, 1995.

[Bon88] Francis Bonahon. The geometry of Teichmüller space via geodesic currents. Invent. Math., 92(1):139-162, 1988.

[Bow99] Brian Bowditch. Treelike structures arising from continua and convergence groups, volume 662. Memoirs Amer. Math. Soc., 1999.

[BV06] Martin R. Bridson and Karen Vogtmann. Automorphism groups of free groups, surface groups and free abelian groups. In Problems on mapping class groups and related topics, volume 74 of Proc. Sympos. Pure Math., pages 301-316. Amer. Math. Soc., Providence, RI, 2006.

[CH14] Thierry Coulbois and Arnaud Hilion. Rips induction : Index of the dual lamination of an $\mathbb{R}$-tree. Groups Geom. Dyn., 8(1):97-134, 2014.

[CHL07] Thierry Coulbois, Arnaud Hilion, and Martin Lustig. Non-unique ergodicity, observers' topology and the dual algebraic lamination for $\mathbb{R}$-trees. Illinois J. Math., 51(3):897-911, 2007. 
[CHL08a] Thierry Coulbois, Arnaud Hilion, and Martin Lustig. $\mathbb{R}$-trees and laminations for free groups. I. Algebraic laminations. J. Lond. Math. Soc. (2), 78(3):723-736, 2008.

[CHL08b] Thierry Coulbois, Arnaud Hilion, and Martin Lustig. $\mathbb{R}$-trees and laminations for free groups. II. The dual lamination of an $\mathbb{R}$-tree. J. Lond. Math. Soc. (2), 78(3):737-754, 2008.

[CHL08c] Thierry Coulbois, Arnaud Hilion, and Martin Lustig. $\mathbb{R}$-trees and laminations for free groups. III. Currents and dual R-tree metrics. J. Lond. Math. Soc. (2), 78(3):755-766, 2008.

[CHL09] Thierry Coulbois, Arnaud Hilion, and Martin Lustig. $\mathbb{R}$-trees, dual laminations, and compact systems of partial isometries. Math. Proc. Cambridge Phil. Soc., 147:345-368, 2009.

[CHR11] Thierry Coulbois, Arnaud Hilion, and Patrick Reynolds. Indecomposable $F_{N}$-trees and minimal laminations. submitted, arXiv:1110.3506, 2011.

[CL95] Marshall M. Cohen and Martin Lustig. Very small group actions on R-trees and Dehn twist automorphisms. Topology, 34(3):575-617, 1995.

[CV86] Marc Culler and Karen Vogtmann. Moduli of graphs and automorphisms of free groups. Invent. Math., 84:91-119, 1986.

[Dur10] Fabien Durand. Combinatorics on Bratteli diagrams and dynamical systems. In Combinatorics, automata and number theory, volume 135 of Encyclopedia Math. Appl., pages 324-372. Cambridge Univ. Press, Cambridge, 2010.

[Gab97] Damien Gaboriau. Générateurs indépendants pour les systèmes d'isométries de dimension un. Ann. Inst. Fourier (Grenoble), 47(1):101-122, 1997.

[GL95] Damien Gaboriau and Gilbert Levitt. The rank of actions on $\mathbb{R}$-trees. Ann. Sci. École Norm. Sup. (4), 28(5):549-570, 1995.

[Gui00] Vincent Guirardel. Dynamics of Out $\left(F_{n}\right)$ on the boundary of outer space. Ann. Sci. École Norm. Sup. (4), 33(4):433$465,2000$.

[Gui08] Vincent Guirardel. Actions of finitely generated groups on $\mathbb{R}$-trees. Ann. Inst. Fourier (Grenoble), 58(1):159-211, 2008.

[Kap05] Ilya Kapovich. The frequency space of a free group. Internat. J. Algebra Comput., 15(5-6):939-969, 2005.

[Kap06] Ilya Kapovich. Currents on free groups. In Topological and asymptotic aspects of group theory, volume 394 of Contemp. Math., pages 149-176. Amer. Math. Soc., Providence, RI, 2006.

[Kea77] Michael Keane. Non-ergodic interval exchange transformations. Israel J. Math., 26(2):188-196, 1977.

[KL07] Ilya Kapovich and Martin Lustig. The actions of Out $\left(F_{k}\right)$ on the boundary of outer space and on the space of currents: minimal sets and equivariant incompatibility. Ergodic Theory Dynam. Systems, 27(3):827-847, 2007.

[KL09] Ilya Kapovich and Martin Lustig. Geometric intersection number and analogues of the curve complex for free groups. Geom. Topol., 13(3):1805-1833, 2009.

[KL10] Ilya Kapovich and Martin Lustig. Intersection form, laminations and currents on free groups. Geom. Funct. Anal., 19(5):1426-1467, 2010.

[KN76] Harvey B. Keynes and Dan Newton. A 'minimal', non-uniquely ergodic interval exchange transformation. Math. Z., 148:101-105, 1976.

[Lev83] Gilbert Levitt. Feuilletages des surfaces. Thèse, Université Paris VII, 1983.

[LL03] Gilbert Levitt and Martin Lustig. Irreducible automorphisms of $F_{n}$ have north-south dynamics on compactified outer space. J. Inst. Math. Jussieu, 2(1):59-72, 2003.

[LL08] Gilbert Levitt and Martin Lustig. Automorphisms of free groups have asymptotically periodic dynamics. J. Reine Angew. Math., 619:1-36, 2008.

[Mas82] Howard Masur. Interval exchange transformations and measured foliations. Ann. of Math. (2), 115(1):169-200, 1982.

[Pap86] Athanase Papadopoulos. Deux remarques sur la géométrie symplectique de l'espace des feuilletages mesurés sur une surface. Ann. Inst. Fourier (Grenoble), 36(2):127-141, 1986.

[Pau88] Frédéric Paulin. Topologie de Gromov équivariante, structures hyperboliques et arbres réels. Invent. Math., 94(1):5380, 1988.

[Sat75] E. A. Sataev. The number of invariant measures for flows on orientable surfaces. Izv. Akad. Nauk SSSR Ser. Mat., 39(4):860-878, 1975.

[Yoc05] Jean-Christophe Yoccoz. Échanges d'intervalles. Cours au Collège de France, 2005. 